\title{
A NOTE ON THE SET OF DISCONTINUITY POINTS OF MULTIFUNCTIONS OF TWO VARIABLES
}

\author{
GraŻYNA KWIECIŃSKA
}

\begin{abstract}
In this paper, we consider some problem connected with separate properties of a multifunction of two variables which ensure that the set of its joint discontinuity points is a meager set.
\end{abstract}

\section{Introduction}

There is a function $f: \mathbb{R}^{2} \rightarrow \mathbb{R}$, whose all $x$-sections $f_{x}(y)=f(x, y)$ and $y$-sections $f^{y}(x)=f(x, y)$ are continuous, and whose set of discontinuity points is of the positive Lebesgue measure [11. However, such a function has always a set of discontinuity points of the first Baire category [17]. On the other hand, if the family $\left\{f_{x}\right\}_{x \in \mathbb{R}}$ is equicontinuous at a point $y_{0}$ and $y_{0}$-section of $f$ is continuous at a point $x_{0}$, then $f$ is continuous at the point $\left(x_{0}, y_{0}\right)$. The problem of finding separate properties of functions such that they have at least one point of joint continuity was widely considered in literature ([1], [4, [6], [8], [9], [16]). However, much less is known about the multivalued case. We will discuss some separate properties of a multifunction of two variables which ensure that the set of its joint discontinuity points is small in a sense of category. Some results of this type connected with quasi-continuity are given by M. Matejdes [15].

\section{Preliminaries}

We begin with a conventional notation. The sets of positive integers and real numbers will be denoted by $\mathbb{N}$ and $\mathbb{R}$, respectively.

(c) 2014 Mathematical Institute, Slovak Academy of Sciences. 2010 Mathematics Subject Classification: 54C60, 54C08 28B20, 26 B05.

Keywords: multifunctions, semicontinuity of a multifunction, lower and upper Baire classes of multifunctions. 


\section{GRAŻYNA KWIECIŃSKA}

Let $S$ and $Z$ be nonempty sets and let $\Phi: S \leadsto Z$ be a multifunction, i.e.,

If $G \subset Z$, then

$$
\emptyset \neq \Phi(s) \subset Z \text { for } s \in S \text {. }
$$

$$
\Phi^{-}(G)=\{s \in S: \Phi(s) \cap G \neq \emptyset\} \quad \text { and } \quad \Phi^{+}(G)=\{s \in S: \Phi(s) \subset G\} .
$$

Since $\Phi^{+}(G) \subset \Phi^{-}(G)$ always, $\Phi^{+}(G)$ and $\Phi^{-}(G)$ are often called small and big inverse images of the set $G$, respectively.

Let $(S, \mathcal{T}(S))$ be a topological space. Then, $\mathcal{B}(S)$ will denote a $\sigma$-field of Borel subsets of $S, \mathcal{B}(s)$ a filterbase of open neighbourhoods of $s \in S, \mathrm{Cl}(A)$, the closure of $A \subset S$ and $\operatorname{Int}(A)$, an interior of $A, \mathcal{P}_{0}(Z)$ a family of all nonempty subsets of $S$, and $\mathcal{C}(Z)$ its subfamily of nonempty compact subsets of $S$.

Various notions of continuity of multifunctions, not equivalent, are known. They all reduce to the usual continuity if a single valued function is considered. We state two different definitions of continuity of multifunctions which we shall use throughout the paper.

Let $(Z, \mathcal{T}(Z))$ be a topological space.

A multifunction $\Phi: S \leadsto Z$ is called lower (resp. upper) semicontinuous at a point $s \in S$, if, for every $G \in \mathcal{T}(Z)$ with $s \in \Phi^{-}(G)\left(s \in \Phi^{+}(G)\right)$, there is $U(s) \in \mathcal{B}(s)$ such that $U(s) \subset \Phi^{-}(G)\left(U(s) \subset \Phi^{+}(G)\right)$. A multifunction $\Phi$ is called lower (resp. upper) semicontinuous, if it is lower (resp. upper) semicontinuous at each point $s \in S$; $\Phi$ is called continuous if it is both lower and upper semicontinuous.

It is easy to see that

(1) $\Phi$ is lower (resp. upper) semicontinuous if and only if $\Phi^{-}(G) \in \mathcal{T}(S)$ (resp. $\Phi^{+}(G) \in \mathcal{T}(S)$ ) for each $G \in \mathcal{T}(Z)$.

If $\Phi: S \leadsto Z$ is a multifunction, then $D_{l}(\Phi)$ and $D_{u}(\Phi)$ will denote the sets of lower discontinuity points and upper discontinuity points of $\Phi$, respectively. Then, $D(\Phi)=D_{l}(\Phi) \cup D_{u}(\Phi)$ is the set of discontinuity points of $\Phi$.

Now, let $\left\{\Phi_{i}\right\}_{i \in \mathcal{I}}$ be a collection of multifunctions $\Phi_{i}: S \leadsto Z, i \in \mathcal{I}$, where $\mathcal{I}$ denotes a set of indexes. A collection of multifunctions $\left\{\Phi_{i}\right\}_{i \in \mathcal{I}}$ is called topologically lower (resp. upper) equicontinuous at a point $s \in S$ if, for for every $G \in \mathcal{T}(Z)$, there exists $U(s) \in \mathcal{B}(s)$ such that $U(s) \subset \Phi_{i}^{-}(G)\left(U(s) \subset \Phi_{i}^{+}(G)\right)$ whenever $s \in \Phi_{i}^{-}(G)\left(s \in \Phi_{i}^{+}(G)\right), i \in \mathcal{I}$. A multifunction $\Phi$ is called topologically lower (resp. upper) equicontinuous, if it is topologically lower (resp. upper) equicontinuous at each point $s \in S ;\left\{\Phi_{i}\right\}_{i \in \mathcal{I}}$ is called topologically eqicontinuous if it is both topologically lower and upper equicontinuous.

In the case when values of a multifunction are subsets of a metric space, we can consider its semicontinuity based on hemimetrics (i.e., pseudometrics which fail to be symmetric) generated by the metric.

Let $(Z, d)$ be a metric space. Let $h_{u}$ and $h_{l}$ denote, respectively, the upper and the lower hemimetric in $\mathcal{P}_{0}(Z)$ generated by the metric $d$, i.e., 
(2) $h_{u}(A, B)=\sup \{d(x, A): x \in B\}$ and $h_{l}(A, B)=\sup \{d(x, B): x \in A\}$.

Note that $h_{l}(A, B)=h_{u}(B, A)$.

A function $h$ on the product $\mathcal{P}_{0}(Z) \times \mathcal{P}_{0}(Z)$ given by

$$
h(A, B)=\max \left\{h_{u}(A, B), h_{l}(A, B)\right\}
$$

is a pseudometric on $\mathcal{P}_{0}(Z)$. Obviously, the space $(\mathcal{C}(Z), h)$ is a metric space.

If $(T, \delta)$ is a hemimetric space, then an open ball will be denoted in the same way as in the case of a metric or a pseudometric. If interior points and open sets are defined in the usual way for hemimetric space $(T, \delta)$, then the family of all open sets is a topology on the space $T$.

In particular, hemimetrics $h_{l}$ and $h_{u}$ generate the lower and the upper hemimetric topology, respectively, on the space $\mathcal{P}_{0}(Z)$.

As usual, $B(z, \varepsilon)$ will denote an open ball of radius $\varepsilon>0$ centred at $z \in Z$. Moreover, if $A \subset Z$, then

$$
B(A, \varepsilon)=\bigcup_{z \in A} B(z, \varepsilon) .
$$

A multifunction $\Phi: S \leadsto Z$ is called $h$-lower (resp. h-upper) semicontinuous at a point $s_{0} \in S$ if for each $\varepsilon>0$ there exists $U\left(s_{0}\right) \in \mathcal{B}\left(s_{0}\right)$ such that $\Phi\left(s_{0}\right) \subset B(\Phi(s), \varepsilon)\left(\Phi(s) \subset B\left(\Phi\left(s_{0}\right), \varepsilon\right)\right)$ for each $s \in U\left(s_{0}\right)$.

A multifunction $\Phi$ is called $h$-lower (resp. h-upper) semicontinuous, if it is $h$-lower (resp. $h$-upper) semicontinuous at each point $s \in S$; $\Phi$ is called $h$-continuous if it is both $h$-lower and $h$-upper semicontinuous.

If $\Phi: S \leadsto Z$ is a multifunction with values in a metric space, then $D_{h-l}(\Phi)$ and $D_{h-u}(\Phi)$ will denote the sets of $h$-lower discontinuity points and $h$-upper discontinuity points of $\Phi$, respectively.

In general, if the values of a multifunction $\Phi$ are subsets of a metric space, then $D_{l}(\Phi) \subset D_{h-l}(\Phi)$ and $D_{h-u}(\Phi) \subset D_{u}(\Phi)$.

It is well-known that if $\Phi$ is compact valued, then the upper (resp. lower) semicontinuity of $\Phi$ and $h$-upper (resp. $h$-lower) semicontinuity of $\Phi$ are equivalent [12. So, in this case, $D_{l}(\Phi)=D_{h-l}(\Phi)$ and $D_{h-u}(\Phi)=D_{u}(\Phi)$.

Now, let $\left\{\Phi_{i}\right\}_{i \in \mathcal{I}}$ be a collection of multifunctions $\Phi_{i}: S \leadsto Z, i \in \mathcal{I}$, where $\mathcal{I}$ denotes a set of indexes. A collection of multifunctions $\left\{\Phi_{i}\right\}_{i \in \mathcal{I}}$ is called h-lower (resp. h-upper) equicontinuous at $s_{0} \in S$ if, for each $\varepsilon>0$, there exists $U\left(s_{0}\right) \in \mathcal{B}\left(s_{0}\right)$ such that $s \in U\left(s_{0}\right)$ implies $\Phi_{i}\left(s_{0}\right) \subset B\left(\Phi_{i}(s), \varepsilon\right)$ (resp. $\Phi_{i}(s) \subset B\left(\Phi_{i}\left(s_{0}\right), \varepsilon\right)$ ) for each $i \in \mathcal{I}$. $\left\{\Phi_{i}\right\}_{i \in \mathcal{I}}$ is called $h$-lower (resp. $h$-upper) equicontinuous if it is $h$-lower (resp. $h$-upper) equicontinuous at each point $s \in S ;\left\{\Phi_{i}\right\}_{i \in \mathcal{I}}$ is called $h$-equicontinuous if it is both $h$-lower and $h$-upper equicontinuous.

In [13], K. K u r a t o w s k i introduced Baire classification of multifunctions. 


\section{GRAŻYNA KWIECIŃSKA}

As before, let $(S, \mathcal{T}(S))$ be a topological space. Given any countable ordinal number $\alpha$, let $\Sigma_{\alpha}(S)$ and $\Pi_{\alpha}(S)$ denote the additive and multiplicative class $\alpha$, respectively, in the Borel hierarchy of subsets of the space $(S, \mathcal{T}(S))$. Following K. K u ratow ski [13, we say that a multifunction $\Phi: S \leadsto Z$ is of the lower (resp. upper) Baire class $\alpha$ if $\Phi^{-}(G) \in \Sigma_{\alpha}(S)$ (resp. $\Phi^{+}(G) \in \Sigma_{\alpha}(S)$ ) whenever $G \in \mathcal{T}(Z)$.

The lower and upper Baire classes of multifunctions of one variable have been extensively studied, among others, by R. B ris a c [2, J. Ewert 3] R. W. Hansel [7] and K. M. Garg [5], where many of the known results on real functions and also on lower and upper semicontinuous multifunctions have been extended to the general Baire classes of multifunctions.

We shall use $L B_{\alpha}$ and $U B_{\alpha}$ to denote a family of multifunctions of the lower (resp. upper) Baire class $\alpha$, respectively.

By (1), it follows that $\Phi \in L B_{0}$ (resp. $\Phi \in U B_{0}$ ) if and only if $\Phi$ is lower (resp. upper) semicontinuous.

As regards the mutual relationship between the lower and upper Baire classes, the following is known (see [5]):

(3) Let $(Z, \mathcal{T}(Z))$ be a perfect topological space (i.e., every open set in $Z$ is

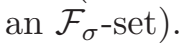

(i) If $\Phi: S \leadsto Z$ is closed valued and $\Phi \in U B_{\alpha}$, then $\Phi \in L B_{\alpha+1}$.

(ii) If, moreover, $(Z, \mathcal{T}(Z))$ is normal, $\Phi$ is compact valued and $\Phi \in L B_{\alpha}$, then $\Phi \in U B_{\alpha+1}$.

The following theorem is known (see [3]).

(4) Let $(Z, \mathcal{T}(Z))$ be a second countable topological space.

(i) If $\Phi: S \leadsto Z$ is of the lower Baire class 1, then the set $D_{l}(\Phi)$ is of the first category.

(ii) If $\Phi: S \leadsto Z$ is compact valued and of the upper Baire class 1, then the set $D_{u}(\Phi)$ is of the first category.

\section{Main results}

Now, we will consider the multifunctions of two variables. Obviously, each multifunction of two variables $x \in X$ and $y \in Y$ may be treated as a multifunction of the single variable $(x, y) \in X \times Y$. The essential difference is the possibility of formulating hypotheses concerning the multifunction in terms of its sectionwise properties.

Let us put $S=X \times Y$ and $\left(x_{0}, y_{0}\right) \in X \times Y$. Let $F: X \times Y \leadsto Z$ be a multifunction. Then, the multifunction $F_{x_{0}}: Y \leadsto Z$ defined by $F_{x_{0}}(y)=F\left(x_{0}, y\right)$ 


\section{A NOTE ON THE SET OF DISCONTINUITY POINTS}

is called $x_{0}$-section of $F$, and the multifunction $F^{y_{0}}: X \leadsto Z$ defined by $F^{y_{0}}(x)=$ $F\left(x, y_{0}\right)$ is called $y_{0}$-section of $F$.

$\mathrm{S} . \mathrm{K}$ e $\mathrm{m}$ p i s t y in 10 has shown that each real function of two real variables upper semicontinuous in the first and lower semicontinuous in the second variable is of the first Baire class. Some multivalued analogy of this theorem is known (see [14, Theorems 1 and 3]).

Theorem 1. Let $(X, \mathcal{T}(X))$ be a topological space, $(Y, \mathcal{T}(Y))$ a metrizable space and $(Z, \mathcal{T}(Z))$ a perfectly normal topological space. If $F: X \times Y \leadsto Z$ is a compact valued multifunction such that for each $(x, y) \in X \times Y$, the section $F_{x}$ is upper semicontinuous and the section $F^{y}$ is lower semicontinuous, then $F$ is of the upper Baire class 1. If, moreover, $(Z, \mathcal{T}(Z))$ is second countable, then $F$ is also of the lower Baire class 1.

As an immediate consequence of the above theorem and (4), we have

Theorem 2. Let $(X, \mathcal{T}(X))$ be a topological space, let $(Y, \mathcal{T}(Y))$ be a metrizable space, and let $(Z, \mathcal{T}(Z))$ be a perfectly normal and second countable topological space. If $F: X \times Y \leadsto Z$ is a compact valued multifunction such that for each $(x, y) \in X \times Y, F_{x}$ is upper semicontinuous and $F^{y}$ is lower semicontinuous, then the sets $D_{u}(F)$ and $D_{l}(F)$ are of the first category and so is the set $D(F)$.

In connection with Theorem 1, let us consider the following example.

Example 1. Decompose $\mathbb{R}$ into two disjoint sets $A \notin \mathcal{B}(\mathbb{R})$ and $B \notin \mathcal{B}(\mathbb{R})$. Define a multifunction $F: \mathbb{R}^{2} \leadsto \mathbb{R}$ putting

$$
F(x, y)= \begin{cases}{[-3,3]} & \text { if } x \neq y, \\ {[1,2]} & \text { if } x=y \in A, \\ {[-1,0]} & \text { if } x=y \in B .\end{cases}
$$

Then,

$F^{+}((0,3))=\{(x, y): F(x, y) \subset(0,3)\}=\{(a, a): a \in A\} \notin \mathcal{B}\left(\mathbb{R}^{2}\right)$

and

$F^{-}((-2,0))=\{(x, y): F(x, y) \cap(-2,0) \neq \emptyset\}=\mathbb{R}^{2} \backslash\{(a, a): a \in A\} \notin \mathcal{B}\left(\mathbb{R}^{2}\right)$.

Moreover, for any open set $G \subset \mathbb{R}$, the sets $F_{x}^{-}(G)$ and $F^{y-}(G)$ are open. So, $F$ is neither of any lower Baire class nor of any upper Baire class even if its sections $F_{x}$ and $F^{y}$ are lower semicontinuous for any $(x, y) \in \mathbb{R}^{2}$.

However, the following theorem is true.

Proposition 1. Let $(X, \mathcal{T}(X))$ and $(Y, \mathcal{T}(Y))$ be topological spaces and let $(Z, d)$ be a metric space. If a multifunction $F: X \times Y \leadsto Z$ is such that $\left\{F_{x}\right\}_{x \in X}$ is h-lower equicontinuous and $F^{y}$ is h-lower semicontinuous for each $y \in Y$, then $F$ is h-lower semicontinuous. 


\section{GRAŻYNA KWIECIŃSKA}

Proof. Fix $\left(x_{0}, y_{0}\right) \in X \times Y$ and $\varepsilon>0$. Since $F^{y_{0}}$ is $h$-lower semicontinuous at $x_{0}$, there is $U\left(x_{0}\right) \in \mathcal{B}\left(x_{0}\right)$ such that

(5) $F\left(x_{0}, y_{0}\right) \subset B\left(F\left(\left(x, y_{0}\right), \frac{\varepsilon}{2}\right)\right)$ for all $x \in U\left(x_{0}\right)$.

By the $h$-lower equicontinuity of $\left\{F_{x}\right\}_{x \in X}$ at $y_{0}$, there is $V\left(y_{0}\right) \in \mathcal{B}\left(y_{0}\right)$ such that

(6) $F\left(x, y_{0}\right) \subset B\left(F\left((x, y), \frac{\varepsilon}{2}\right)\right)$ for all $x \in X$ and $y \in V\left(y_{0}\right)$.

Let $W\left(x_{0}, y_{0}\right)=U\left(x_{0}\right) \times V\left(y_{0}\right)$. Then, by (5) and (6),

$$
F\left(x_{0}, y_{0}\right) \subset B(F((x, y), \varepsilon)) \quad \text { for } \quad(x, y) \in W\left(x_{0}, y_{0}\right),
$$

i.e., $F$ is $h$-lower semicontinuous at $\left(x_{0}, y_{0}\right)$.

The next theorem is a consequence of Proposition 1 and theorems (3) (ii) and (4) (ii).

TheOREM 3. Let $(X, \mathcal{T}(X))$ and $(Y, \mathcal{T}(Y))$ be topological spaces and let $(Z, d)$ be a separable metric space. Let $F: X \times Y \leadsto Z$ be a compact valued multifunction. If $\left\{F_{x}\right\}_{x \in X}$ is topologically lower equicontinuous and for every $y \in Y$ the section $F^{y}$ is lower semicontinuous, then the set $D_{u}(F)$ is of the first category and so is the set $D(F)$.

The following has an almost identical proof to that of Proposition 1.

Proposition 2. Let $(X, \mathcal{T}(X))$ and $(Y, \mathcal{T}(Y))$ be topological spaces and let $(Z, d)$ be a metric space. If a multifunction $F: X \times Y \leadsto Z$ is such that $\left\{F_{x}\right\}_{x \in X}$ is h-upper equicontinuous and $F^{y}$ is h-upper semicontinuous for each $y \in Y$, then $F$ is h-upper semicontinuous.

Similarly, as a consequence of Proposition 2 and theorems (3) (i) and (4) (i), we have

TheOREM 4. Let $(X, \mathcal{T}(X))$ and $(Y, \mathcal{T}(Y))$ be topological spaces and let $(Z, d)$ be a separable metric space. Let $F: X \times Y \leadsto Z$ be a compact valued multifunction. If $\left\{F_{x}\right\}_{x \in X}$ is topologically upper equicontinuous and for every $y \in Y$ the section $F^{y}$ is upper semicontinuous, then the set $D_{l}(F)$ is of the first category and so is the set $D(F)$.

There is a possibility of some variation in Theorem 3 and Theorem 4 .

TheORem 5. Let $(X, \varrho)$ be a separable metric space, $(Y, \rho)$ a complete metric space, and $(Z, d)$ a metric space. If a multifunction $F: X \times Y \leadsto Z$ is such that $\left\{F_{x}\right\}_{x \in X}$ is h-equicontinuous and, for each $y \in Y$, the set $D_{h-l}\left(F^{y}\right)$ is nowhere dense, then the set $D_{h-l}(F)$ is nowhere dense. 


\section{A NOTE ON THE SET OF DISCONTINUITY POINTS}

P r o o f. To derive a contradiction, suppose that the set $D_{h-l}(F)$ is not nowhere dense. Then, there is an open set $W \subset X \times Y$ such that $D_{h-l}(F)$ is dense in $W$. Let $U \subset X$ and $V \subset Y$ be nonempty open sets such that $U \times V \subset W$. By separability of $X$, we choose a family $\left\{B_{n}\right\}_{n \in \mathbb{N}}$ of all nonempty open subsets of $X$ such that $B_{n} \subset U$ for any $n \in \mathbb{N}$. Let $\left(x_{1}, y_{1}\right) \in B_{1} \times V$ be such that $F$ is not $h$-lower semicontinuous at $\left(x_{1}, y_{1}\right)$. Then,

(7) there is $\varepsilon>0$ such that for each $W\left(x_{1}, y_{1}\right) \in \mathcal{B}\left(\left(x_{1}, y_{1}\right)\right)$ there is a point $(x, y) \in W\left(x_{1}, y_{1}\right)$ such that $h_{l}\left(F\left(x_{1}, y_{1}\right), F(x, y)\right) \geq \frac{\varepsilon}{2}$.

By $h$-equicontinuity of $\left\{F_{x}\right\}_{x \in X}$ at $y_{1}$,

(8) for a given $\varepsilon$, there is an open neighbourhood $V\left(y_{1}\right) \in \mathcal{B}\left(y_{1}\right)$ such that $\mathrm{Cl}\left(V\left(y_{1}\right)\right) \subset V$, and, for any $x \in X$ and any $y \in V\left(y_{1}\right)$, we have $h\left(F\left(x, y_{1}\right)\right.$, $F(x, y))<\frac{\varepsilon}{8}$.

Then, $h_{l}\left(F\left(x, y_{1}\right), F(x, y)\right)<\frac{\varepsilon}{8}$ for any $x \in X$ and any $y \in V\left(y_{1}\right)$, and in particular, for $x=x_{1}$,

(9) $h_{l}\left(F\left(x_{1}, y_{1}\right), F\left(x_{1}, y\right)\right)<\frac{\varepsilon}{8}$ for each $y \in V\left(y_{1}\right)$,

and for $x=a_{n}$,

(10) $h_{l}\left(F\left(a_{n}, y_{1}\right), F\left(a_{n}, y\right)\right)<\frac{\varepsilon}{8}$ for each $n \in \mathbb{N}$ and $y \in V\left(y_{1}\right)$.

Moreover, $b_{n} \in V\left(y_{1}\right)$ for $n \in \mathbb{N}$, therefore,

(11) $h_{l}\left(F\left(a_{n}, y_{1}\right), F\left(a_{n}, b_{n}\right)\right)<\frac{\varepsilon}{8}$ for each $n \in \mathbb{N}$.

From (8), it also follows that $h_{l}\left(F(x, y), F\left(x, y_{1}\right)\right)<\frac{\varepsilon}{8}$ for any $x \in X$ and any $y \in V\left(y_{1}\right)$. In particular, for $x=a_{n}$, we have

(12) $h_{l}\left(F\left(a_{n}, y\right), F\left(a_{n}, y_{1}\right)\right)<\frac{\varepsilon}{8}$ for each $n \in \mathbb{N}$ and $y \in V\left(y_{1}\right)$.

By $(7)$, we can choose a sequence $\left(a_{n}, b_{n}\right)_{n \in \mathbb{N}}$ such that

(13) $\lim _{n \rightarrow \infty}\left(a_{n}, b_{n}\right)=\left(x_{1}, y_{1}\right), a_{n} \in B_{1}, b_{n} \in V\left(y_{1}\right)$ and

$$
h_{l}\left(F\left(x_{1}, y_{1}\right), F\left(a_{n}, b_{n}\right)\right) \geq \frac{\varepsilon}{2} \quad \text { for each } \quad n \in N \text {. }
$$

Since

$$
h_{l}\left(F\left(a_{n}, y\right), F\left(a_{n}, b_{n}\right)\right) \leq h_{l}\left(F\left(a_{n}, y\right), F\left(a_{n}, y_{1}\right)\right)+h_{l}\left(F\left(a_{n}, y_{1}\right), F\left(a_{n}, b_{n}\right)\right) \text {, }
$$

by (12) and (11), we have

(14) $h_{l}\left(F\left(a_{n}, y\right), F\left(a_{n}, b_{n}\right)\right) \leq \frac{\varepsilon}{4}$ for each $n \in N$ and $y \in V\left(y_{1}\right)$.

Since, moreover,

$$
h_{l}\left(F\left(x_{1}, y\right), F\left(a_{n}, b_{n}\right)\right) \geq h_{l}\left(F\left(x_{1}, y_{1}\right), F\left(a_{n}, b_{n}\right)\right)-h_{l}\left(F\left(x_{1}, y_{1}\right), F\left(x_{1}, y\right)\right),
$$

from (13) and (9) it follows that

(15) $h_{l}\left(F\left(x_{1}, y\right), F\left(a_{n}, b_{n}\right)\right) \geq \frac{3}{8} \varepsilon$ for each $n \in N$ and $y \in V\left(y_{1}\right)$. 


\section{GRAŻYNA KWIECIŃSKA}

Finally,

$$
h_{l}\left(F\left(x_{1}, y\right), F\left(a_{n}, y\right)\right) \geq h_{l}\left(F\left(x_{1}, y\right), F\left(a_{n}, b_{n}\right)\right)-h_{l}\left(F\left(a_{n}, y\right), F\left(a_{n}, b_{n}\right)\right),
$$

and by (15) and (14), we have

$$
h_{l}\left(F\left(x_{1}, y\right), F\left(a_{n}, y\right)\right) \geq \frac{\varepsilon}{8} \quad \text { for each } \quad n \in \mathbb{N} \text { and } y \in V\left(y_{1}\right) .
$$

Since $\lim _{n \rightarrow \infty} a_{n}=x_{1}$,

(16) $F$ is not $h$-lower semicontinuous at $\left(x_{1}, y\right)$ for each $y \in V\left(y_{1}\right)$.

Now, let us suppose that $\left(x_{2}, y_{2}\right) \in B_{2} \times V\left(y_{1}\right)$ and $F$ is not $h$-lower semicontinuous at $\left(x_{2}, y_{2}\right)$. Similarly as in (16), we can select $V\left(y_{2}\right) \in \mathcal{B}\left(y_{2}\right)$ such that $\mathrm{Cl}\left(V\left(y_{2}\right)\right) \subset V\left(y_{1}\right)$ and $F$ is not $h$-lower semicontinuous at $\left(x_{2}, y\right)$ for each $y \in V\left(y_{2}\right)$. Thus, by induction, taking a point $\left(x_{n}, y_{n}\right) \in B_{n} \times V\left(y_{n-1}\right)$ such that $F$ is not $h$-lower semicontinuous at $\left(x_{n}, y_{n}\right)$, we can select $V\left(y_{n}\right) \in \mathcal{B}\left(y_{n}\right)$ such that

(i) $\mathrm{Cl}\left(V\left(y_{n}\right)\right) \subset V\left(y_{n-1}\right)$ for each $n \in N$ and

(ii) $F$ is not $h$-lower semicontinuous at $\left(x_{n}, y\right)$ for each $y \in V\left(y_{n}\right)$.

Let $y_{0} \in \bigcap_{n \in \mathbb{N}} \mathrm{Cl} V\left(y_{n}\right)$. Then, by (17), $F^{y_{0}}$ is not $h$-lower semicontinuous at $x_{n}$ for each $n \in \mathbb{N}$. The set $\left\{x_{n}\right\}_{n \in \mathbb{N}}$ is dense in $U$ and it is a subset of $D_{h-l}\left(F^{y_{0}}\right)$. Now, we have the required contradiction because, by assumption, the set $D_{h-l}\left(F^{y_{0}}\right)$ is nowhere dense.

A similar proof works when we replace " $h$-lower" with " $h$-upper" in Theorem 5 , and we have a dual result.

TheOREM 6. Let $(X, \varrho)$ be a separable metric space, $(Y, \rho)$ a complete metric space and $(Z, d)$ a metric space. If a multifunction $F: X \times Y \leadsto Z$ is such that $\left\{F_{x}\right\}_{x \in X}$ is h-equicontinuous and for each $y \in Y$ the set $D_{h-u}\left(F^{y}\right)$ is nowhere dense, then the set $D_{h-u}(F)$ is nowhere dense.

It is easy to show the following property.

Proposition 3. Let $(X, \mathcal{T}(X)),(Y, \mathcal{T}(Y))$ and $(Z, \mathcal{T}(Z))$ be topological spaces. Let $F: X \times Y \leadsto Z$ be a multifunction such that $\left\{F_{x}\right\}_{x \in X}$ is topologically lower equicontinuous. Then, $F$ is lower semicontinuous at a point $\left(x_{0}, y_{0}\right) \in X \times Y$ if and only if the section $F^{y_{0}}$ is lower semicontinuos at the point $x_{0}$.

TheOREM 7. Let $(X, \mathcal{T}(X))$ be a second countable topological space, $(Y, \mathcal{T}(Y))$ a topological space and $(Z, \mathcal{T}(Z))$ a second countable topological space. Let $F: X \times Y \leadsto Z$ be a multifunction such that $\left\{F_{x}\right\}_{x \in X}$ is topologically lower equicontinuous and for each $y \in Y$ the set $D_{l}\left(F^{y}\right)$ is of the first category. If for each $G \in \mathcal{T}(Z)$ the set $F^{-}(G)$ has the Baire property, then the set $D_{l}(F)$ is of the first category. 


\section{A NOTE ON THE SET OF DISCONTINUITY POINTS}

Proof. Note that, by the definition, $F$ is not lower semicontinuous at a point $\left(x_{0}, y_{0}\right)$ if and only if $\left(x_{0}, y_{0}\right) \in F^{-}(G)$ and $\left(x_{0}, y_{0}\right) \notin \operatorname{Int}\left(F^{-}(G)\right)$ for some $G \in \mathcal{T}(Z)$. Let $\mathcal{B}=\left\{B_{n}\right\}_{n \in \mathbb{N}}$ be a base of $Z$. Then

$$
D_{l}(F)=\bigcup_{n \in \mathbb{N}}\left(F^{-}\left(B_{n}\right) \backslash \operatorname{Int}\left(F^{-}\left(B_{n}\right)\right)\right) .
$$

Therefore the set $D_{l}(F)$ has the Baire property and from Proposition 3, it follows that it is of the first category.

Like in the case of Proposition 3, it is easy to show the dual proposition.

Proposition 4. Let $(X, \mathcal{T}(X)),(Y, \mathcal{T}(Y))$ and $(Z, \mathcal{T}(Z))$ be topological spaces. Let $F: X \times Y \leadsto Z$ be a multifunction such that $\left\{F_{x}\right\}_{x \in X}$ is topologically upper equicontinuous. Then, $F$ is upper semicontinuous at a point $\left(x_{0}, y_{0}\right) \in X \times Y$ if and only if the section $F^{y_{0}}$ is upper semicontinuos at the point $x_{0}$.

In a similar fashion to that of Theorem 7, we prove a dual result.

TheOREM 8. Let $(X, \mathcal{T}(X))$ be a second countable topological space, $(Y, \mathcal{T}(Y))$ a topological space, and $(Z, \mathcal{T}(Z))$ a second countable topological space. Let $F: X \times Y \leadsto Z$ be a compact valued multifunction such that $\left\{F_{x}\right\}_{x \in X}$ is topologically upper equicontinuous and, for each $y \in Y$, the set $D_{u}\left(F^{y}\right)$ is of the first category. If for each $G \in \mathcal{T}(Z)$ the set $F^{+}(G)$ has the Baire property, then the set $D_{u}(F)$ is of the first category.

P r o of. According to the definition, $F$ is not upper semicontinuous at a point $\left(x_{0}, y_{0}\right)$ if and only if $\left(x_{0}, y_{0}\right) \in F^{+}(G)$ and $\left(x_{0}, y_{0}\right) \notin \operatorname{Int}\left(F^{+}(G)\right)$ for some $G \in \mathcal{T}(Z)$. Let $\mathcal{B}$ be a countable base of $Z$. Since $F\left(x_{0}, y_{0}\right) \subset G$, by compactness, the set $F\left(x_{0}, y_{0}\right)$ is a subset of a finite union of sets from $\mathcal{B}$.

Let $\mathcal{G}$ be a family of all finite unions of elements from $\mathcal{B}$. Then $\mathcal{G}$ is also countable. We can enumerate $\mathcal{G}$ by $\left\{G_{1}, G_{2}, \ldots, G_{n}, \ldots\right\}$. Then,

$$
D_{u}(F)=\bigcup_{k \in \mathbb{N}}\left(F^{+}\left(G_{k}\right) \backslash \operatorname{Int}\left(F^{+}\left(G_{k}\right)\right)\right) \text {. }
$$

Therefore the set $D_{u}(F)$ has the Baire property and from Proposition 4, it follows that it is of the first category.

\section{REFERENCES}

[1] BAIRE, R.: Sur les fonctions des variables réelles, Ann. Mat. Appl. 3 (1899), 1-22.

[2] BRISAC, R.: Les classes de Baire des fonctions multiformes, C. R. Acad. Sci. Paris 224 (1974), 257-258.

[3] EWERT, J.: Multivalued mappings and bitopological spaces. Słupsk, 1985. (In Polish) 


\section{GRAŻYNA KWIECIŃSKA}

[4] FUDALI, L. A.: On cliquish functions on product spaces, Math. Slovaca 33 (1988), $53-58$.

[5] GARG, K. M.: On the classification of set-valued functions, Real Anal. Exchange 9 (1983-1984), 86-93.

[6] GRANDE, Z.: On functions of two variables equicontinuous in one variable, Real Anal. Exchange 22 (1996-97), 760-765.

[7] HANSEL, R. W.: Hereditarily additive families in descriptive set theory and measurable multimaps, Trans. Amer. Math. Soc. 278 (1983), 725-749.

[8] HENRIKSEN, H.-WOODS, R. G.: Separate versus joint continuity: A tale of four topologies, Topology Appl. 97 (1999), 175-205.

[9] HOLÁ, L'.-PIOTROWSKI, Z.: Set of continuity points of functions with values in generalized metric spaces, Tatra Mt. Math. Publ. 42 (2009), 1-12.

[10] KEMPISTY, S.: Sur les fonctions semi-continues par raport á chacume de deux variables, Fund. Math. 14 (1929), 237-241.

[11] KERSHNER, R.: The continuity of functions of many variables, Trans. Amer. Math. Soc. 53 (1943), 83-100.

[12] KLEIN, E.-THOMPSON, A. C.: Theory of Correspondences, in: Canad. Math. Soc. Ser. Monographs Adv. Texts, A Wiley-Interscience Publication. John Wiley \& Sons, Inc., New York, 1984

[13] KURATOWSKI, K.: On set-valued B-measurable mappings and a theorem of Hausdorff, in: Theory Sets Topology, Collection Papers Honour Felix Hausdorff, Berlin, 1972, pp. 355-362.

[14] KWIECIŃSKA, G.: On the Borel class of multivalued functions of two variables, Topology Proc. 25 (2000), 601-613.

[15] MATEJDES, M.: Continuity of multifunctions, Real Anal. Exchange 19 (1992-1993), 394-413.

[16] NEUBRUNN, T.: Quasi-continuity, Real Anal. Exchange 14 (1988-89), 259-305.

[17] PIOTROWSKI, Z.: The genesis of separate versus joint continuity, Tatra Mt. Math. Publ. 8 (1996), 113-126.

Institute of Mathematics

Pomeranian University

Arciszewskiego 22 d

PL-76-200 Stupsk

E-mail: grazyna.kwiecinska@apsl.edu.pl 